\title{
UNLINKING VIA SIMULTANEOUS CROSSING CHANGES
}

\author{
MARTIN SCHARLEMANN
}

\begin{abstract}
Given two distinct crossings of a knot or link projection, we consider the question: Under what conditions can we obtain the unlink by changing both crossings simultaneously? More generally, for which simultaneous twistings at the crossings is the genus reduced? Though several examples show that the answer must be complicated, they also suggest the correct necessary conditions on the twisting numbers.
\end{abstract}

Let $L$ be an oriented link in $S^{3}$ with a generic projection onto the plane $R^{2}$. Let $\alpha$ be a short arc in $R^{2}$ transverse to both strands of $L$ at a crossing, so that the strands pass through $\alpha$ in opposite directions. Then the inverse image of $\alpha$ contains a disk punctured twice, with opposite orientation, by $L$. Define a crossing disk $D$ for a link $L$ in $S^{3}$ to be a disk which intersects $L$ in precisely two points, of opposite orientation. It is easy to see that any crossing disk arises in the manner described. Twisting the link $q$ times as it passes through $D$ is equivalent to doing $1 / q$ surgery on $\partial D$ in $S^{3}$ and adds $2 q$ crossings to this projection of $L$. We say that this new link $L(q)$ is obtained by adding $q$ twists at $D$. Call $\partial D$ a crossing circle for $L$. A crossing disk $D$, and its boundary $\partial D$, are essential if $\partial D$ bounds no disk disjoint from $L$. This is equivalent to the requirement that $L$ cannot be isotoped off $D$ in $S^{3}-\partial D$.

Here we examine how a link can be turned into the unlink via simultaneous twists on disjoint crossing disks. In particular, we prove an analogue for pairs of crossing disks of the following theorem, a much more general version of which is proven in [ST, 1.4] (see also [Ga]):

0.1. Theorem. If $D$ is an essential crossing disk for the unlink $L$, then the link obtained by adding $q \neq 0$ twists to $L$ at $D$ is not the unlink.

A moment's reflection suggests problems in finding an analogue for pairs of disks. Consider some examples:

0.2. Example. Suppose $K_{1}$ and $K_{2}$ are two essential crossing circles for any link $L$, and suppose that $K_{1}$ and $K_{2}$ are unlinked in $S^{3}$ and bound an annulus in $S^{3}-L$. Then adding $q$ twists at $K_{1}$ and $-q$ twists at $K_{2}$ leaves $L$ unchanged. In particular, if $L$ was the unlink before the twists were added, it will be the unlink afterwards.

Received by the editors January 21, 1991.

1991 Mathematics Subject Classification. Primary 57M25; Secondary 57M40.

The author was supported in part by a National Science Foundation grant. 


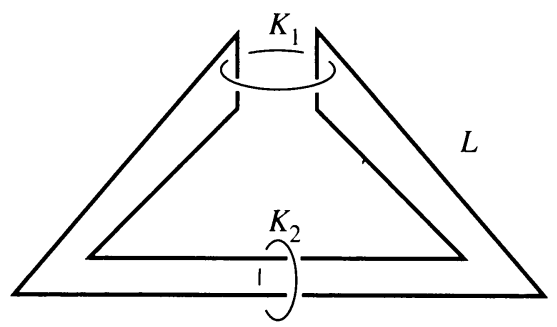

FIGURE 1

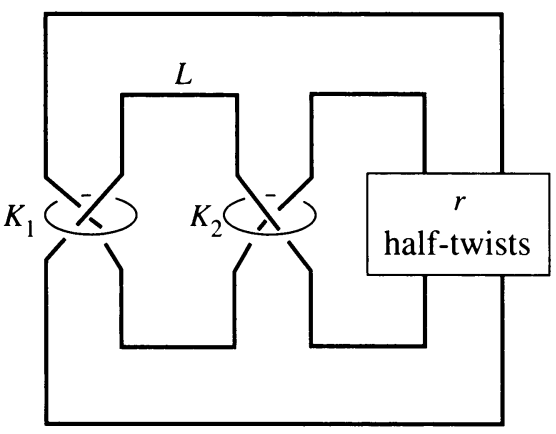

FIGURE 2

To see this, let $D$ be a crossing disk for $K_{1}$. Using the annulus $A$ we can find an imbedding $D \times I$ in $S^{3}$ so that $L \cap(D \times I)=(L \cap D) \times I$ and $D \times \partial I$ is the union of crossing disks at $K_{1}$ and $K_{2}$. Then the operation of adding $q$ twists at one end and adding $-q$ twists at the other is clearly isotopic to the identity.

0.3. Example. Let $K_{1}$ and $K_{2}$ be crossing disks for the unknot, as shown in Figure 1. Then in general adding $p$ twists at $K_{1}$ and $q$ twists at $K_{2}$ gives a twist knot. But if either $p=0$ or $q=0$, then $L$ is the unlink. Note here that $K_{1}$ (resp. $\left.K_{2}\right)$ is inessential if and only if $q=0$ (resp. $\left.p=0\right)$.

0.4. Example. Let $K_{1}$ and $K_{2}$ be crossing disks for the unknot $L$, as shown in Figure 2, with $r$ odd. Then adding $p$ twists at $K_{1}$ and $q$ twists at $K_{2}$ gives the pretzel knot of type $(2 p+1,2 q-1, r)$. According to [Bo; BZ, 12D], $L$ is the unknot if and only if two of the three numbers $\{2 p+1,2 q-1, r\}$ have opposite sign and absolute value 1 . Thus, if $r \neq \pm 1, L$ is the unknot if and only if $(p, q)=(-1,1)$ or $(0,0)$. If $r=1$, then $L$ is the unlink if and only if either $p=-1$ or $q=0$. If $r=-1$, then $L$ is the unlink if and only if either $p=0$ or $q=1$.

Example 0.4 suggests that the general solution could be quite complicated. In fact, 0.4 points to a general statement. We say that a finite set of disjoint crossing circles $\left\{K_{i}\right\}$ for $L$ is an essential set of crossing circles if no $K_{i}$ bounds a disk in $S^{3}-\left(L \cup\left(\bigcup_{j} K_{j}\right)\right)$. To deal with the problem raised by Example 0.2, define a pair $\partial D_{1}, \partial D_{2}$ of crossing circles for $L$ to be coannular if there is an annulus $A$, disjoint from $L$, such that $\partial A \cap D_{i}=\partial D_{i}$. Given $L$ and a pair $K_{1}, K_{2}$ of crossing circles for $L$, let $L\left(t_{1}, t_{2}\right)$ be the link obtained from $L$ by adding $t_{1}$ twists at $K_{1}$ and $t_{2}$ twists at $K_{2}$. 
0.5. Theorem. Let $K_{1}, K_{2}$ be an essential pair of noncoannular crossing disks for a link $L \subset S^{3}$. Then there is a pair $\left(s_{1}, s_{2}\right)$ of integers such that whenever $L\left(t_{1}, t_{2}\right)$ is the unlink, either $t_{1}=s_{1}$ or $t_{2}=s_{2}$.

Clearly if $L$ itself is the unlink then one of the $s_{i}$ must be 0 . In Example 0.3 when $r \neq \pm 1$, we can take for $\left(s_{1}, s_{2}\right)$ either $(-1,0)$ or $(0,1)$. When $r=1$, take $\left(s_{1}, s_{2}\right)=(-1,0)$; when $r=-1$ take $\left(s_{1}, s_{2}\right)=(0,1)$.

Theorem 0.5 is a consequence of a more general result, closer in spirit to [ST, 1.4]. Recall that a link in a 3-manifold is split if it is isotopic to the distant union of two sublinks. A link $L$ has splitting number $n$ if $L$ is isotopic to the distant union of $n$ nonsplittable sublinks. Suppose $S$ is an orientable surface. Following Thurston [Th], define $\chi_{-}(S)$ to be $-\chi(C)$, where $C$ is the union of nonsimply connected components of $S$. For $L$ a link in $S^{3}$, define $\chi_{-}(L)$ to be the minimal value of $\chi_{-}(S)$ for all oriented incompressible surfaces $S$ in $S^{3}$ with $\partial S=L$. Let $K_{1}$ and $K_{2}$ be boundaries of disjoint crossing circles for a link $L$ in $S^{3}$. $L$ bounds an orientable surface $S$ disjoint from $K=K_{1} \cup K_{2}$. Define $\chi_{K}(L)$ to be the minimal value of $\chi_{-}(S)$ for all oriented incompressible surfaces $S$ in $S^{3}-K$ with $\partial S=L$. Let $L\left(t_{1}, t_{2}\right)$ denote the link obtained from $L$ by adding $t_{1}$ twists at $K_{1}$ and $t_{2}$ twists at $K_{2}$. A pair $\left(t_{1}, t_{2}\right)$ of integers is norm-reducing for $L$ at $K$ if either the splitting number of $L\left(t_{1}, t_{2}\right)$ in $S^{3}$ is greater than that of $L$ in $S^{3}-\left(K_{1} \cup K_{2}\right)$ or $\chi_{-}\left(L\left(t_{1}, t_{2}\right)\right)<\chi_{K}(L)$. We then have:

2.3. Theorem. Let $K=K_{1} \cup K_{2}$ be an essential pair of noncoannular crossing disks for a link $L \subset S^{3}$. Then there is a pair $\left(s_{1}, s_{2}\right)$ of integers so that for every norm-reducing pair $\left(t_{1}, t_{2}\right)$ for $L$ at $K$, either $t_{1}=s_{1}$ or $t_{2}=s_{2}$.

It is easy to see that Theorem 0.5 follows from Theorem 2.3 . The only singlecomponent link of trivial norm is the unknot. So if a link $L$ in $S^{3}-K$ splits in $S^{3}-K$ into single component knots, each of trivial norm, then $L$ bounds a collection of disks in $S^{3}-K$ and $K$ is not an essential pair for $L$. Thus either $L$ does not completely split in $S^{3}-K$ or one of its components is knotted and it has nontrivial norm. Hence, if $L\left(t_{1}, t_{2}\right)$ is the unlink, $\left(t_{1}, t_{2}\right)$ is norm-reducing.

The outline is as follows: In $\S 1$ we treat what is apparently a very special case, that of links which lie on the boundary of a genus two handlebody. In $\S 2$ sutured manifold theory and [Sc2] are applied to the proof of 2.3 , hence 0.5 . It is shown that in fact $\S 1$ treated the critical case. There is also a Property P type theorem about surgery on strongly invertible pairs of knots. Section 3 contains a generalization to Dehn fillings on pairs of tori in arbitrary orientable compact 3-manifolds.

Much of $\S 3$ was prompted by a very helpful conversation with Abby Thompson.

\section{A SPECIAL CASE-LINKS ON A HANDLEBODY}

Let $H$ be a genus two handlebody in $S^{3}$ with specified meridian disks $\mu_{1}$ and $\mu_{2}$. Let $\Gamma \subset \partial H$ be an oriented collection of disjoint simple closed curves such that each curve of $\Gamma$ intersects at least one meridian and each meridian is either disjoint from $\Gamma$ or intersects $\Gamma$ twice, with opposite orientation. Let $\Gamma\left(t_{1}, t_{2}\right)$ denote the curves obtained from $\Gamma$ by twisting $t_{1}$ times around 


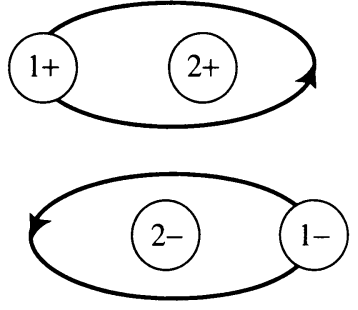

case 1

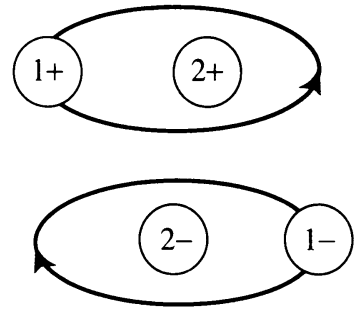

case 2

Figure 3

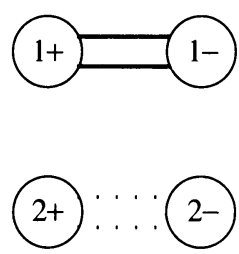

case 3
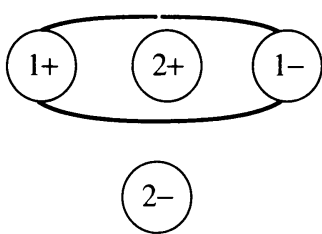

case 4
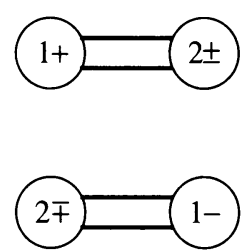

case 5

FIGURE 4

meridian $\mu_{1}$ and $t_{2}$ times around meridian $\mu_{2}$. Let $X$ denote the closure of $S^{3}-H$. Let $\Phi$ be the set of pairs $\left(t_{1}, t_{2}\right)$ such that some component of $\Gamma\left(t_{1}, t_{2}\right)$ bounds a disk in $X$.

\subsection{Lemma. One of the following holds:}

(a) There is a pair $\left(s_{1}, s_{2}\right)$ in $\Phi$ such that $\Phi=\left\{\left(t_{1}, t_{2}\right) \mid t_{1}=s_{1}\right.$ or $\left.t_{2}=s_{2}\right\}$.

(b) There is an $s_{1}$ such that $\Phi=\left\{\left(t_{1}, t_{2}\right) \mid t_{1}=s_{1}\right\}$.

(c) There is an $s_{2}$ such that $\Phi=\left\{\left(t_{1}, t_{2}\right) \mid t_{2}=s_{2}\right\}$.

(d) $\Phi$ has exactly two elements $\left(t_{1}, t_{2}\right),\left(t_{1}^{\prime}, t_{2}^{\prime}\right)$ with $\left|t_{i}-t_{i}^{\prime}\right|=1, i=1,2$.

(e) $\Phi$ has at most one element.

Proof. Let $Q$ denote the 4-punctured sphere $\partial H-\left\{\mu_{i}\right\}$. We may assume that the 1-manifold $\gamma=\Gamma \cap Q$ consists of oriented essential arcs in $Q$. The four components of $\partial Q$ may be labelled $\mu_{i}^{ \pm}, i=1,2$, in the obvious manner. We may assume that $\Gamma \cap \mu_{1}$ contains two points. If there is a single arc of $\gamma$ with ends at $\mu_{1}^{ \pm}$, say, then, since any arc of $\gamma$ is essential in $Q, \gamma$ must be one of the configurations in Figure 3.

If the two arcs of $\gamma$ having ends on $\mu_{1}^{+}$have their other end on the same component of $\partial Q$, then $\gamma$ must be one of the three configurations of Figure 4.

If the two arcs of $\gamma$ having ends on $\mu_{1}^{+}$have their other end on different components of $\partial Q$ then $\gamma$ must be one of the two configurations in Figure 5.

In cases 3 and 4 in Figure 4 , there is a component $k_{1}$ of $\Gamma$ which intersects $\mu_{1}$ once and is disjoint from $\mu_{2}$. Similarly, either $\Gamma$ is disjoint from $\mu_{2}$ or there is a component $k_{2}$ intersecting $\mu_{2}$ once but not $\mu_{1}$. At most one choice of $t_{1}$ allows $k_{1}$ to bound a disk in $S^{3}-H$ since two different choices have nontrivial intersection number in $\partial H$. Similarly, at most one choice of $t_{2}$ allows $k_{2}$ to bound a disk in $S^{3}-H$. If some such $t_{1}$ and $t_{2}$ exist we have case (a). If a choice of $t_{1}$ but not $t_{2}$ exists (or vice versa) we have case (b) (or (c)) of 1.1. If no choice of either exists, we have case (e). 


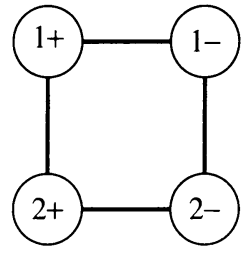

case 6

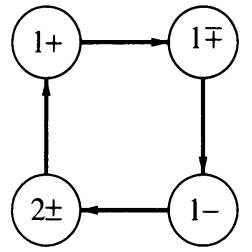

case 7

FIGURE 5

Almost the same argument finishes case 6 of Figure 5; again we have the components $k_{1}$ and $k_{2}$, but also a component $k_{3}$ intersecting both meridia exactly once. Suppose there is a choice of $t_{1}$ after which $k_{1}$ bounds a disk in $S^{3}-H$. Then just as before, $k_{3}$ cannot bound a disk after any twisting along $\mu_{1}$, since its algebraic intersection with the original $k_{1}$ would be nontrivial. Similarly for $t_{2}$ and $k_{2}$. So the situation is the same as above. On the other hand, suppose there is no choice of $t_{1}$ or $t_{2}$ allowing $k_{1}$ or $k_{2}$ to bound disks. Then we may as well discard them and consider only $k_{3}$. This is equivalent to case 5 of Figure 4, treated below.

To treat the diagrams in cases $1,2,5$, and 7 , a bit more is needed. Suppose a component $k$ of $\Gamma=\Gamma(0,0)$ and a component $k^{\prime}$ of $\Gamma\left(t_{1}, t_{2}\right)$ bound disks $D$ and $D^{\prime}$ respectively. Minimize $\partial D \cap \partial D^{\prime}$ by isotoping across any bigons of $k \cup k^{\prime}$ bounding disks in $\partial H$. A standard innermost disk argument allows us to assume that $D$ and $D^{\prime}$ intersect in arcs. An outermost arc of $D^{\prime} \cap D$ in $D^{\prime}$ cuts off a subdisk $F$ along which $D$ may be compressed to give two disks $E$ and $E^{\prime}$, both disjoint from $D$. Both $E$ and $E^{\prime}$ are compressing disks for $\partial H$ in $S^{3}-H$ which are disjoint from $D$. In sum, we have the following algorithm: Examine successive intersection points of $k^{\prime}$ with $k$. Let $\alpha^{\prime}$ be the subarc of $k^{\prime}$ lying between them, so $\alpha^{\prime} \cap k=\varnothing$. Let $\alpha$ be either arc of $k$ lying between the intersection points. Then $\alpha \cup \alpha^{\prime}$ is a simple closed curve in $H$ which is disjoint from $k$. At least two curves constructed in this manner bound disks in $X$.

Apply this algorithm to the remaining diagrams: Applied to diagrams 1 or 2 it gives a simple closed curve in $Q$ which is parallel to $\mu_{2}$ and compresses in $S^{3}-H$. Applied to diagram 5, the algorithm gives a simple closed curve in $Q$ which separates $\mu_{1}^{+}$from $\mu_{1}^{-}$and which compresses in $X$. Neither is possible. hence we have case $(e)$.

When the algorithm is applied to diagram 7 we are only able to conclude that there is a compressing disk $E$ in $X$ so that $E$ is disjoint from $D$ and passes at most once over each meridian.

Case 1: $\partial E$ intersects both meridians. In diagram 7, $\partial E$ appears as two arcs, parallel either to the vertical or horizontal arcs of $\partial D$ in the diagram, say the former. Then a circle in $S^{2}$ separating the right two disks from the left two is disjoint from $\partial E$ and intersects $k=\partial D$ in two points. Denote by $\mu_{3}$ the disk this circle bounds in $H$. Then $\mu_{3}$ is a meridian of the solid torus $K$ obtained from $H$ by attaching a 2-handle with core $E$.

Subcase (i): $K$ is unknotted. This is essentially Example 0.4 above. Both $k$ and $k^{\prime}$ are pretzel knots, with an odd number of half-twists in each band. $H$ 
can be viewed as a regular neighborhood of the natural Seifert surfaces of these pretzel knots. Such a Seifert surface consists of two disks connected by three bands, each dual to one of the $\mu_{i}$ and each having an odd number of halftwists. Let $h_{i}$ [resp. $h_{i}^{\prime}$ ] denote the number of half-twists in the band dual to $\mu_{i}, i=1,2,3$. Then $k$ is unknotted if and only if $h_{i}=-h_{j}= \pm 1$ for some $i \neq j$ [Bo; BZ, 12D]. If $\pm 1=-h_{3}=h_{1}$ or $h_{2}$, then there is a compressing disk in $X$, disjoint from $k$, intersecting $\mu_{1}$ or $\mu_{2}$ exactly once. This case is essentially Case (2) below. The case in which $\pm 1=-h_{3}=h_{1}^{\prime}$ or $h_{2}^{\prime}$ is similar, with the roles of $k$ and $k^{\prime}$ switched. Finally, if $h_{1}=-h_{2}= \pm 1$ and $-h_{1}^{\prime}=h_{2}^{\prime}= \pm 1$, then $h_{1}$ and $h_{1}^{\prime}$, and $h_{2}$ and $h_{2}^{\prime}$ each differ from each other by a single full twist. This is case (d) above.

Subcase (ii): $K$ is knotted. Then all compressing disks for knots in $K$ may be taken to lie in a neighborhood of $K$. Thus, at the risk of introducing possibly new elements of $\Phi$, we may replace $K$ by the unknot, hence $k$ with a pretzel knot as above, with $h_{3}$ chosen to be large. This forces, at best, case (d). But we observe that in case (d) above the compressions of $k$ and $k^{\prime}$ do indeed take place in a neighborhood of $K$, so we have introduced no new elements to $\Phi$.

Case (2): $\partial E$ intersects only one meridian. Say $\partial E$ is disjoint from $\mu_{1}$, and intersects $\mu_{2}$ once. We have $\Gamma(0,0)=k(0,0)=k$ and will show that either (c) or (a) holds with $s_{2}=0$. If $E$ were disjoint from $\mu_{2}$ and intersected $\mu_{1}$, then we would have (b) or (a) with $s_{1}=0$.

First we show that any $(\cdot, 0)$ lies in $\Phi$. Let $K$ denote the solid torus with meridian $\mu_{1}$ obtained from $H$ by attaching a 2-handle along $E$. Then $k \subset \partial K$ bounds a disk in $S^{3}-K$ and is null-homologous in $K$, so it is trivial in $\partial K$. Any $k(\cdot, 0)$ is disjoint from $\partial E$ so it lies in $\partial K$, and differs from $k$ by some twists around the meridian of $K$. Hence any $k(\cdot, 0)$ also bounds a disk in $\partial K$, so it bounds a disk in $X$.

On the other hand, let $\left(t_{1}, t_{2}\right)$ be any nontrivial element of $\Phi$, so $k^{\prime}=$ $k\left(t_{1}, t_{2}\right)$ bounds a disk $D^{\prime}$ in $X$. We wish to determine $\left(t_{1}, t_{2}\right)$. The band sum of $E$ to itself along $\mu_{1}$ gives a separating disk $F$ for $X$ with $\partial F$ disjoint from $\mu_{1}$ and $\mu_{2} . F$ splits $X$ into the boundary connect sum of two knot complements, one the unknot with longitude $\partial E$ and the other $S^{3}-K$. The curve $\partial F$ intersects $k^{\prime}$ in four points, so an outermost arc of $F \cap D^{\prime}$ in $D^{\prime}$ cuts off a disk from $D^{\prime}$ which is a longitude of one of the two knots, either $\partial E$ or a longitude of $K$. In the former case, $t_{2}=0$. In the latter, $K$ is the unknot and $t_{1}$ must be the specific slope $s_{1}$ for which some arc cut off from $k^{\prime}$ by $\partial F$ is a longitude of $K$. Thus if $K$ is knotted we have (c), and if $K$ is unknotted we have $(\mathbf{a})$.

\section{SUTURES IN GENUS TWO SURFACES}

2.1. Definition. Suppose $M$ is a compact orientable 3-manifold $M$ and $P \subset$ $\partial M$ is a possibly disconnected closed surface. We say $M$ is a $J$-cobordism on $P$ if $H_{2}(M, \partial M-P)=0$.

2.2. Lemma. If $M$ is a $J$-cobordism on $P$, then genus $(\partial M-P) \leq \operatorname{genus}(P)$. Proof. Let $Q=\partial M-P$. Consider the following commutative diagram induced by inclusion: 


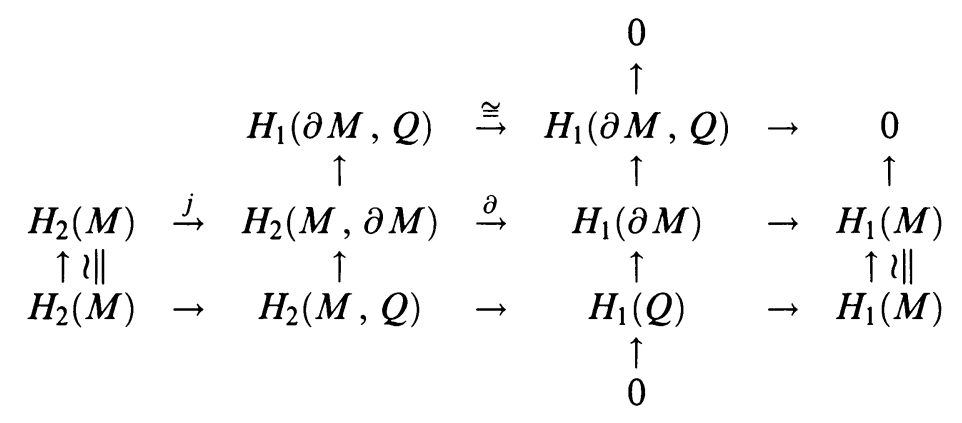

Using the fact that $H_{2}(M, Q)=0$ and $\partial M-Q=P$ this simplifies to:

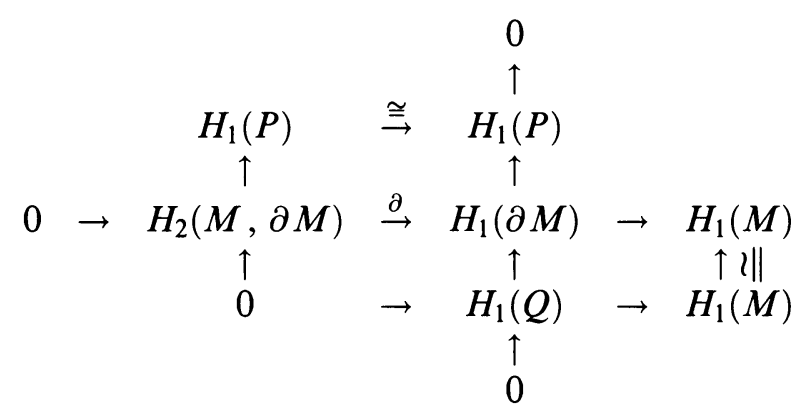

Let $p=\operatorname{rank} H_{1}(P), q=\operatorname{rank} H_{1}(Q)$, and $s=\operatorname{rank} H_{2}(M, \partial M)$. A standard argument from Poincaré duality shows $2 s=\operatorname{rank} H_{1}(\partial M)=p+q$. From the left-most vertical arrows we get $s \leq p$. Hence $q \leq p$.

2.3. Theorem. Let $K=K_{1} \cup K_{2}$ be an essential pair of noncoannular crossing disks for a link $L \subset S^{3}$. Then there is a pair $\left(s_{1}, s_{2}\right)$ of integers so that for every norm-reducing pair $\left(t_{1}, t_{2}\right)$ for $L$ at $K$, either $t_{1}=s_{1}$ or $t_{2}=s_{2}$.

Proof. An innermost circle argument shows that any essential sphere in $S^{3}-$ $(K \cup L)$ can be isotoped off of a pair $D=D_{1} \cup D_{2}$ of crossing disks bounded by $K$. If any essential sphere separates $K_{1}$ from $K_{2}$, then the theorem naturally follows from separate applications of [ST, 1.4] to $K_{1}$ and $K_{2}$. So suppose no essential sphere separates $K_{1}$ from $K_{2}$. Then any essential sphere in $S^{3}-$ $(L \cup D)$ splits off a sublink distant from $D$ which is unaffected by twists at $D$, and so may be ignored. So we may henceforth assume that $S^{3}-(K \cup L)$ is irreducible.

Let $\eta(K)$ and $\eta(L)$ denote tubular neighborhoods of $K$ and $L$ respectively, and let $M$ be the irreducible manifold $S^{3}-\eta(K \cup L)$. Let $T_{i} \subset \partial M$ be the torus $\partial \eta\left(K_{i}\right)$, and let $\lambda_{i}$ and $\mu_{i}$ denote the longitude and meridian of $\partial \eta\left(K_{i}\right)$ respectively. Let $S \subset M$ be an incompressible surface so that $\partial S=L$ and $\chi_{-}(S)$ is as low as possible, so $\chi_{-}(S)=\chi_{K}(L)$.

Regard $(M, \partial M)$ as a sutured manifold (cf. [Sc1]). Construct a taut sutured manifold hierarchy

$$
(M, \eta(L)) \stackrel{S_{1}}{\longrightarrow}\left(M_{1}, \gamma_{1}\right) \stackrel{S_{2}}{\longrightarrow} \cdots \stackrel{S_{i}}{\longrightarrow}\left(M_{i}, \gamma_{i}\right) \stackrel{S_{i+1}}{\longrightarrow} \cdots
$$

so that $S_{1}=S$, and each $S_{i}$ is disjoint from $T=T_{1} \cup T_{2}$. By [ $\mathrm{Sc}_{1}, 2.6$ and 4.17] such a sequence can be extended as long as $H_{2}\left(M_{i}, \partial M_{i}-T\right) \neq 0$, but eventually 
terminates in a sutured manifold $\left(M_{n}, \gamma_{n}\right)$ with $H_{2}\left(M_{n}, \partial M_{n}-T\right)=0$, i.e., a $J$-cobordism on $T$. Then by 2.2 , genus $\left(\partial M_{n}-T\right) \leq 2$.

The crossing disks become a pair of 2-punctured disks in $M$. Regard $D \cap M$ as a parametrizing surface for the hierarchy [Sc1, $\S 7]$. Consider the effect of the decompositions in the hierarchy on a component of $D \cap M$. Take, for example, $Q=D_{1} \cap M . Q$ is a pair of pants, with two boundary components meridia of $L$ and the other $\lambda_{1}$. Since $\chi(Q)=-1, Q$ has index 2 , and so its remnant $Q_{n}$ in $M_{n}$ is a planar surface of index no more than 2 .

2.4. Lemma. Some component of $Q_{n}$ is an annulus $A$ with one boundary component $\lambda_{1}$ and the other a simple closed curve on $\partial M_{n}-T$ which intersects the set of sutures at most twice.

Proof. First we show that some component is an annulus from $\lambda_{1}$ to a curve in $\partial M_{n}-T$. This part is by induction in the sutured manifold hierarchy. We begin by observing that the initial Seifert surface $S$ may be taken to intersect $Q$ in a single arc connecting the two punctures (see [ST]), so $Q_{1}$ is an annulus from $\lambda_{1}$ to a circle crossing the suture $\partial \eta(L)-\partial S$ exactly twice. Suppose inductively that $Q_{i}$ has an annular component $A$ with one end $\lambda_{1}$ and the other end lying on $\partial M_{i}-T$. Examine the 1 -manifold $\Gamma=S_{i+1} \cap A$. Since $S_{i+1}$ is disjoint from $T$, no arc of $\Gamma$ has an end on $\lambda_{1}$. Since $S_{i+1}$ is incompressible, we may assume that any closed component is essential in $A$. If there is a closed component, then the one nearest to $\lambda_{1}$ in $A$ cuts off the required annulus from $A$. If there are no closed components, then all components are arcs with neither end on $\lambda_{1}$, so the component of $A-S_{i+1}$ containing $\lambda_{1}$ remains an annulus.

It remains to check that the final annulus $A \subset Q_{n}$ intersects sutures in $\partial M_{n}-$ $T$ no more than twice. Since $Q_{n}$ is a parametrizing surface, no component of $Q_{n}$ can have negative index, and each component must have even index, since each boundary component crosses the sutures an even number of times. The total index is no more than index $Q_{1}=2$. Hence $Q_{n}$ can have no more than one component of positive index, and it must have index 2. In particular, $A$ can have index at most 2 , so $\partial A$ intersects the set of sutures in $\partial M_{n}-T$ at most twice.

To continue the proof of Theorem 2.3, assume that $\left(M_{n}, \gamma_{n}\right)$ is taut, and that $H_{2}\left(M_{n}, \partial M_{n}-T\right)=0$. Let $(\widetilde{M}, \gamma)$ denote the sutured manifold obtained from $\left(M_{n}, \gamma_{n}\right)$ by filling back in $\eta\left(K_{i}\right)$. More generally, let $(\widetilde{M}, \gamma)\left(t_{1}, t_{2}\right)$ denote the sutured manifold obtained from $\left(M_{n}, \gamma_{n}\right)$ by filling in a solid torus along $\partial \eta\left(K_{i}\right)$ with slope $1 / t_{i}$, that is, by attaching solid tori so that the meridian of each torus is homologous to $t_{i} \lambda_{i}+\mu_{i}$.

From 2.4 (applied also to $Q=D_{2} \cap M$ ), we know there are annuli $A_{i}$ in $M_{n}$, each with one end on $\lambda_{i}$ and with the other a curve $c_{i}$ in $\partial M_{n}-T$ intersecting $\gamma_{n}$ at most twice. We can assume that $\left|c_{i} \cap \gamma_{n}\right|$ cannot be reduced by an isotopy of $c_{i}$. Each $c_{i}$ is essential in $\partial M_{n}$ since $T$ is incompressible, and the $c_{i}$ are not parallel in $\partial M_{n}$ since $K_{1}$ and $K_{2}$ are not coannular in $M_{n} \subset M$. We know that genus $\left(\partial M_{n}-T\right) \leq 2$; since $\partial M_{n}-T$ contains the two nonparallel inessential circles $c_{i}$ we have that genus $\left(\partial M_{n}-T\right)=2$. That is, the component(s) of $\partial M_{n}-T$ containing the $c_{i}$ consist either of a single genus 2 surface, or two tori.

Since the meridian of the filling torus at $\eta\left(K_{i}\right)$ intersects $\lambda_{i}$ once, there is 
a natural homeomorphism of $\widetilde{M}$ to $M_{n}-\eta\left(A_{1} \cup A_{2}\right)$ under which $c_{i}$ corresponds to a curve parallel to $\lambda_{i}$ in $M_{n}$. In fact, then, the underlying manifolds of $(\widetilde{M}, \gamma)$ and $(\widetilde{M}, \gamma)\left(t_{1}, t_{2}\right)$ are the same; the only difference is that in $(\widetilde{M}, \gamma)\left(t_{1}, t_{2}\right)$ the sutures $\gamma$ have been altered by Dehn twists on the curves $c_{i}$. Hence we will write $\left(\widetilde{M}, \gamma\left(t_{1}, t_{2}\right)\right)$ instead of $(\widetilde{M}, \gamma)\left(t_{1}, t_{2}\right)$.

2.5. Lemma. $\widetilde{M}$ is irreducible. If $\left(\widetilde{M}, \gamma\left(t_{1}, t_{2}\right)\right)$ is not taut, then some suture in $\gamma\left(t_{1}, t_{2}\right)$ bounds a disk in $\widetilde{M}$.

Proof. Without loss of generality, take $\left(t_{1}, t_{2}\right)=(0,0)$ so $\left(\widetilde{M}, \gamma\left(t_{1}, t_{2}\right)\right)=$ $(M, \gamma)$. Any sphere in $\widetilde{M}$ can be pushed along the annuli $A_{i}$ and across the filling tori so that it lies in $M_{n}$. Since $\left(M_{n}, \gamma_{n}\right)$ is taut, $M_{n}$ is irreducible, so the sphere bounds a ball in $M_{n} \subset \widetilde{M}$. Hence $\widetilde{M}$ is irreducible.

$\chi_{-}(\partial \widetilde{M})=0$ or 2 . Let $R_{ \pm}$denote the two submanifolds into which $\gamma$ divides $\partial \widetilde{M}$. Then $\chi_{-}\left(R_{+}\right)=\chi_{-}\left(R_{-}\right)=\frac{1}{2} \chi_{-}(\partial \widetilde{M})=0$ or 1 . Hence each of $R_{ \pm}$consists of a union of annuli and one other component, either a punctured torus or a 3-punctured sphere. There are two possible ways in which $(\widetilde{M}, \gamma)$ could fail to be taut:

Suppose $\gamma$ bounds a surface $R^{\prime}$ in $\widetilde{M}$ with $\chi_{-}\left(R^{\prime}\right)<\chi_{-}\left(R_{ \pm}\right)$. Then $\chi_{-}\left(R^{\prime}\right)=0$ and $\chi_{-}\left(R_{ \pm}\right)=1$. Since $\chi_{-}\left(R_{ \pm}\right)=1, \gamma$ has an odd number of components. The only way a surface with an odd number of boundary components can have trivial $\chi_{-}$is if one component is a disk.

Suppose some component of $R_{ \pm}$is compressible. If the component is not an annulus, then the previous case applies. If it is an annulus, then after compression it becomes disks.

Remark. Lemma 2.5 requires genus $(\partial \widetilde{M}) \leq 2$ and thereby limits the methods here to twists on at most two crossing circles.

2.6. Lemma. There is a pair $\left(s_{1}, s_{2}\right)$ of integers so that if $\left(\widetilde{M}, \gamma\left(t_{1}, t_{2}\right)\right)$ is not taut, then either $t_{1}=s_{1}$ or $t_{2}=s_{2}$.

Proof. There are two cases to consider:

Case (1): $\partial \widetilde{M}$ contains two torus components, $U_{1}$ and $U_{2}$, with $c_{i} \subset U_{i}$. If $\gamma$ does not intersect $c_{i}$, then it is unaffected by Dehn twists at $c_{i}$. No component of $\gamma \cap U_{i}$ disjoint from $c_{i}$ can bound a disk in $\widetilde{M}$, since $\left(M_{n}, \gamma_{n}\right)$ is taut. All sutures in $\gamma \cap U_{i}$ which intersect $c_{i}$ are parallel. If $\gamma_{0}$ is such a suture, and $\gamma_{1}$ is the image of $\gamma_{0}$ by a Dehn twist along $c_{i}$, then $\gamma_{0} \cdot \gamma_{1} \neq 0$, so at most one of them can bound a disk. Hence there is at most one twisting $t_{1}$ of $c_{1}$ and $t_{2}$ of $c_{2}$ after which $\left(\widetilde{M}, \gamma\left(t_{1}, t_{2}\right)\right)$ is not taut.

Before considering the other case, consider this. Since we only care if a suture in $\gamma$ bounds a disk in $\widetilde{M}$, we may reimbed $\widetilde{M}$ in $S^{3}$ with no effect on the problem. Excision of $\eta\left(A_{1} \cup A_{2}\right)$ shows the first homomorphism in the composition $H_{2}\left(M_{n}, \partial M_{n}-T\right) \rightarrow h_{2}\left(\widetilde{M}, \partial \widetilde{M}-\left(c_{1} \cup c_{2}\right)\right) \rightarrow H_{2}(\widetilde{M}, \partial \widetilde{M})$ is surjective. Since $H_{2}\left(M_{n}, \partial M_{n}-T\right)=0$, the second homomorphism must be trivial. Then for any component $W$ of $\widetilde{M}$, there is an imbedding of $W$ in $S^{3}$ so that $S^{3}-W$ is a union of handlebodies $H$, and $C=c_{1} \cup c_{2}$ contains a complete system of meridia for $H$ [Sc2]. 
Case (2): There is a genus two component $U$ of $\partial \widetilde{M}_{n}$ containing $C$. Let $W$ be the component of $\widetilde{M}_{n}$ such that $U \subset \partial W$. Any other component of $\partial W$ must be a sphere, since genus $(\partial \widetilde{M})=2$. Since $\widetilde{M}$ is irreducible, we conclude that $\partial W=U$.

We have seen that we may take $S^{3}-W$ to be a genus two handlebody $H$, on which $C$ is a pair of meridia. The sutures $\gamma$ intersect each $c_{i}$ at most twice. No suture disjoint from the $c_{i}$ compresses in $W$, for if it did, then it would compress in $M_{n}$, and $\left(M_{n}, \gamma_{n}\right)$ is taut. The proof in this case now follows formally from 1.1. In cases (a) and (e) the choice of $s_{1}$ and $s_{2}$ is obvious. In case (b) (or (c)), the choice of $s_{1}$ (or $s_{2}$ ) is given, and the other is arbitrary. In case (d), take $s_{1}=t_{1}$ and $s_{2}=t_{2}^{\prime}$. Lemma 2.6 is proved.

The proof of Theorem 2.3 is now immediate from Lemma 2.6 and [ $\mathrm{Sc} 1$, 3.9].

We give a modest "property P" type of application:

2.7. Definition. Let $L=L_{1} \cup L_{2} \subset S^{3}$ be a link of two components. $L$ is strongly invertible if there is an involution of $S^{3}$ taking each $L_{i}$ to itself, but reversing its orientation.

2.8. Proposition. Suppose $L=L_{1} \cup L_{2}$ is strongly invertible, $L_{1}$ and $L_{2}$ are not coannular in $S^{3}$, and neither component bounds a disk in the complement of the other. Let $M\left(p_{1}, p_{2}\right)$ be the manifold obtained from $S^{3}$ by surgery on $L$ with slope $1 / 2 p_{i}$ on each $L_{i}$. Then for one of the links $L_{i}$ and some integer $q \neq 0$, if $M\left(p_{1}, p_{2}\right)$ is simply connected then either $p_{1}=p_{2}=0$ or $p_{i}=q$.

Proof. The proof is modelled on [BS, 1.7]. Since $L$ is strongly invertible, the involution on $S^{3}$ must be rotation about an unknotted axis $A$ which pierces each $L_{i}$ twice. The axis $A$ projects to a branch set $B$, which is the unknot in $S^{3}$, and each $L_{i}$ projects to an arc $\alpha_{i}$ with ends on $B$.

The involution on $S^{3}$ induces an involution on $M\left(p_{1}, p_{2}\right)$. If $M\left(p_{1}, p_{2}\right)$ is simply connected, then the branch set $B^{\prime}$ for this action must be the unknot in $S^{3}$ [B]. There is a crossing circle $K_{i}$ for $B$ on the boundary of a regular neighborhood of $\alpha_{i}$ that lifts to a longitude of $L_{i}$, and $B^{\prime}$ can be viewed as having been obtained from $B$ by adding $p_{i}$ twists at $K_{i}, i=1,2$ (cf. [Mo, Li]). If $K=K_{1} \cup K_{2}$ is not an essential pair, then one of the $L_{i}$ bounds a disk in the complement of the other. If $K_{1}$ and $K_{2}$ were coannular in $S^{3}-B$, then the lift of the annulus would make $L_{1}$ and $L_{2}$ coannular in $S^{3}$. Now apply Theorem 0.5. There is a pair of integers $\left(q_{1}, q_{2}\right)$ such that $B^{\prime}$ is unknotted if and only if $p_{1}=q_{1}$ or $p_{2}=q_{2}$. Since $B$ is unknotted, one of the $q_{i}$, say $q_{2}$, is trivial. Thus if $B^{\prime}$ is unknotted either $p_{1}=q_{1}$ or $p_{2}=0$. But if $p_{2}=0$, then $p_{1}=0[\mathrm{BS}]$.

Remark. Note that for $\left(1 / 2 p_{1}, 1 / 2 p_{2}\right)$ surgery to give a homology 3 -sphere it is necessary that $L_{1}$ and $L_{2}$ have trivial algebraic linking. The above proposition can be generalized to surgeries with slopes of the form $\left(2 r+1 / 2 p_{1}, 2 s+1 / 2 p_{2}\right)$ by making a different choice of crossing circles $K_{i}$ near the arcs $\alpha_{i}$. This might be useful in that cases where $L_{1}$ and $L_{2}$ have nontrivial algebraic linking number. 


\section{A genERALIZATION}

3.1. Definition. Suppose $M$ is a 3-manifold and $T$ is a torus component of $\partial M$. Let $\sigma$ be an essential simple closed curve in $T$. Then there is a homeomorphism $\varphi: \partial D^{2} \times S^{1} \rightarrow T$, well defined up to isotopy, such that $\varphi\left(\partial D^{2}\right)=\sigma$. The manifold $M(\sigma)$ obtained by attaching $D^{2} \times S^{1}$ to $M$ via $\varphi$ is a filling of $M$ at $T$ with slope $\sigma . D^{2} \times S^{1} \subset M(\sigma)$ is called the filling torus and $\{0\} \times S^{1}$ is called the core of the filling torus. If $\sigma$ and $\tau$ are two essential simple closed curves in $T$, isotoped to minimize $|\sigma \cap \tau|$, then $|\sigma \cap \tau|$ is denoted $\sigma \cdot \tau$ and is called the difference in slope between $\sigma$ and $\tau$.

Recall the following theorem, a reformulation of $[\mathrm{Ga}, 1.8]$.

3.2. Theorem. Let $(M, \gamma)$ be a connected taut sutured manifold with $\gamma \neq \varnothing$, and $T \subset \partial M$ a torus such that $\gamma \cap T=\varnothing$. Suppose the only $J$-cobordism on $T$ contained in $M$ is $T \times I$. Then there is at most one slope $\sigma$ for which $(M(\sigma), \gamma)$ is not taut.

Subsequent discoveries now allow a variant of this theorem, with the hypothesis on $J$-cobordisms removed, and the conclusion only slightly weakened:

3.3. Theorem. Let $(M, \gamma)$ be a connected taut sutured manifold with $\gamma \neq \varnothing$, and $T \subset \partial M$ a torus such that $\gamma \cap T=\varnothing$. Then there are at most three slopes $\sigma$ for which $(M(\sigma), \gamma)$ is not taut. Indeed, if $\sigma$ and $\tau$ are such exceptional slopes, then $\sigma \cdot \tau \leq 1$. At most one of the exceptional $M(\sigma)$ is irreducible.

Proof. Special case: $M$ is a J-cobordism on $T$ such that $\partial M-T$ is a torus $T^{\prime}$. Since $(M, \gamma)$ is taut, all the sutures $\gamma$ are essential in $T^{\prime}$, thus are parallel essential curves. Now $(M(\sigma), \gamma)$ can fail to be taut for two reasons: either $M(\sigma)$ is reducible, or the annuli $T^{\prime}-\gamma$ compress in $M(\sigma)$. The latter can happen for at most one slope $\sigma$.

If $T^{\prime}-\gamma$ compresses in $M(\sigma)$ for slope $\sigma$, and $M\left(\sigma^{\prime}\right)$ is reducible for slope $\sigma^{\prime}$, then it follows from [Sc3, 6.1] that the core of the filling torus in $M(\sigma)$ is a cabled knot $k$ in $M(\sigma)$ and $\sigma^{\prime}$ is the slope of a cabling annulus $A^{\prime}$. In particular, $\sigma \cdot \sigma^{\prime}=1$. Suppose there were another slope $\sigma^{\prime \prime}$ for which $M\left(\sigma^{\prime \prime}\right)$ is reducible. Then the slope $\sigma^{\prime \prime}$ is that of another cabling annulus $A^{\prime \prime}$ for $k$. A simple combinatorial argument on the intersection of $A^{\prime}$ and $A^{\prime \prime}$ shows this contradicts, for example, the irreducibility of $M$. Hence only the fillings $\sigma$ and $\sigma^{\prime}$ can possibly produce nontaut sutured manifolds.

Suppose now that $T^{\prime}-\gamma$ does not compress in any $M(\sigma)$, so the only way in which $(M(\sigma), \gamma)$ can fail to be taut is if $M(\sigma)$ is reducible. Gordon and Luecke [GL] have shown that any two fillings of $T$ which produce reducible 3-manifolds differ in slope by at most one.

General case: The proof in general follows from the special case just as in [Ga, 1.8]: If $M$ itself is not a $J$-cobordism on $T$, then one can construct a taut sutured manifold hierarchy of $(M, \gamma)$, using always surfaces disjoint from $T$, until we reach a sutured manifold of the form $\left(M_{n}, \gamma_{n}\right)$ with $M_{n}$ a $J$-cobordism on $T$. Then, by $2.2, \partial M_{n}-T$ has genus at most one. Since $(M, \gamma)$ is taut, $M$ is irreducible, so any sphere boundary component of $M_{n}$ must bound a ball. Since $M$ is connected, the component $W$ of $M_{n}$ containing $T$ must have other boundary components, hence its boundary is the union of $T$ and another torus $T^{\prime}$ containing sutures. As in [Ga, 1.8], $(M(\sigma), \gamma)$ is taut 
if the sutured manifold obtained from $W$ by filling with slope $\sigma$ at $T$ is taut. The proof now follows from the special case applied to $W$.

Below we shall present a theorem for simultaneous fillings on the union of two tori $T=T_{1} \cup T_{2}$ which combines features of both 3.2 and 3.3. We begin with some remarks about $J$-cobordisms on tori.

3.4. Definition. Suppose $W$ is a $J$-cobordisms on a torus $T$ such that $\partial W-$ $T$ is a torus $T^{\prime}$. Then $W$ is called a $J$-cobordism between $T$ and $T^{\prime}$.

3.5. Lemma. Suppose $W$ is a J-cobordism on a torus $T$ such that $\partial W-T$ is a torus $T^{\prime}$. Then $W$ is also a J-cobordism on $T^{\prime}$.

There is an intersection-pairing preserving isomorphism

$$
\theta: H_{1}(T, Q) \rightarrow H_{1}\left(T^{\prime}, Q\right)
$$

defined by the requirement that $\theta(\alpha)$ is homologous to $\alpha$ in $H_{1}(W, Q)$.

Proof. This is an elementary consequence of Poincare duality. See, for example, $[\mathrm{Ga}, 1.5]$.

3.6. Definition. A $J$-cobordism as in 3.5 is called a $J$-cobordism between $T$ and $T^{\prime}$.

3.7. Lemma. Let $W$ be a $J$-cobordism between tori $T$ and $T^{\prime}$. Suppose $\mu$ and $\lambda$ are simple closed curves in $T^{\prime}$ so that $\mu \cdot \lambda=1$. Suppose $\sigma$ and $\tau$ are fillings at $T$ so that $\mu$ compresses in $W(\sigma)$ and $\lambda$ compresses in $W(\tau)$. Then $\theta(\mu)= \pm \sigma, \theta(\lambda)= \pm \tau$, and $\sigma \cdot \tau=1$.

Proof. Let $D_{\mu}$ be a compressing disk for $\mu$ in $W(\sigma)$. Then $D_{\mu} \cap W$ is a homology between $\mu$ and $\omega \sigma$, where $\omega$ is an integer. Hence $\theta(\mu)=\omega \sigma$. Similarly, $\theta(\lambda)=\omega^{\prime} \tau$ for some integer $\omega^{\prime}$. Then $\mu \cdot \lambda=\theta(\mu) \cdot \theta(\lambda)=\left|\omega \omega^{\prime}\right| \sigma \cdot \tau$. Since $\mu \cdot \lambda=1,|\omega|=\left|\omega^{\prime}\right|=\sigma \cdot \tau=1$.

As in 3.2, a simplifying assumption about $J$-cobordisms on $T$ in $M$ will be needed: A $J$-cobordism $W$ on $T=T_{1} \cup T_{2}$ is split if it is either the disjoint union or the boundary connected sum of $J$-cobordisms $W_{1}$ on $T_{1}$ and $W_{2}$ on $T_{2}$.

3.8. Definition. Let $(M, \gamma)$ be a taut connected sutured manifold and $T=$ $T_{1} \cup T_{2} \subset \partial M$ the union of tori $T_{1}$ and $T_{2}$ such that $\gamma \cap T=\varnothing$. Let $\left(M\left(\sigma_{1}, \sigma_{2}\right), \gamma\right)$ denote the sutured manifold obtained from $(M, \gamma)$ by filling $T_{i}$ with slope $\sigma_{i}$. Then we say the pair $\left(\sigma_{1}, \sigma_{2}\right)$ is norm-reducing for $M$ if $\left(M\left(\sigma_{1}, \sigma_{2}\right), \gamma\right)$ is not taut.

3.9. Theorem. Let $(M, \gamma)$ be a connected taut sutured manifold with $\gamma \neq \varnothing$, and $T=T_{1} \cup T_{2} \subset \partial M$ the union of tori $T_{1}$ and $T_{2}$ such that $\gamma \cap T=\varnothing$. Suppose any $J$-cobordism on $T$ contained in $M$ is split. Then there are slopes $\tau_{1}$ and $\tau_{2}$ such that for any norm-reducing slope pair $\left(\sigma_{1}, \sigma_{2}\right)$ for $M$, either $\sigma_{1} \cdot \tau_{1} \leq 1$ or $\sigma_{2} \cdot \tau_{2} \leq 1$.

If any $M\left(\sigma_{1}, \sigma_{2}\right)$ is reducible, so is $M\left(\tau_{1}, \tau_{2}\right)$.

Note that if no $M\left(\sigma_{1}, \sigma_{2}\right)$ is reducible, the theorem does not claim that $\left(\tau_{1}, \tau_{2}\right)$ is necessarily norm-reducing for $M$.

Proof. Special case: $M$ itself is a split $J$-cobordism on $T$. If $M$ is the disjoint union of $J$-cobordisms on $T_{1}$ and $T_{2}$, just apply 3.3. 
Suppose $M$ is the boundary connected sum of $J$-cobordisms $W_{1}$ on $T_{1}$ and $W_{2}$ on $T_{2}$ along a disk $E$. Since $M$ is irreducible, no boundary component is a sphere. Then $\partial M-T$ is a genus two surface $F$. Let $F_{1}$ and $F_{2}$ denote the punctured tori into which $\partial E$ divides $F$. Then $\partial M=T_{1} \cup T_{2} \cup F$, and $F=F_{1} \cup_{\partial E} F_{2}$.

Consider the $J$-cobordism $W_{1}$ on $T_{1}, \gamma \cap F_{1}$ is a set of disjoint essential arcs and circles in $F_{1}$. Let $\delta_{1}$ be the set of simple closed curves in the torus $F_{1} \cup E$ obtained by connecting the ends of each essential arc of $\gamma \cap F_{1}$ by an arc across $E$. Two curves in $\delta_{1}$ intersect in at most one point.

Similarly define a set $\delta_{2}$ of simple closed curves in the torus $F_{2} \cup E \subset \partial W_{2}$.

Rule 1 . If there is a filling $\sigma$ of $T_{i}$ which makes $W_{i}$ reducible take $\tau_{i}=\sigma$.

Rule 2. If $W_{i}$ remains irreducible after any filling of $T_{i}$, but there is a slope $\sigma$ of $T_{i}$ such that some curve in $\delta_{i}$ compresses in $W_{i}(\sigma)$, take $\tau_{i}$ to be one such slope $\sigma$.

Rule 3. If none of the $\delta_{i}$ compress and $W_{i}$ remains irreducible after any filling of $T$, take $\tau_{i}$ to be any slope at all.

Suppose $\left(\sigma_{1}, \sigma_{2}\right)$ is a norm-reducing slope pair.

If $M\left(\sigma_{1}, \sigma_{2}\right)$ is reducible then an innermost disk argument on the intersection of a reducing sphere with $E$ shows that either $W_{1}\left(\sigma_{1}\right)$ or $W_{2}\left(\sigma_{2}\right)$ is reducible. Then by [GL] and Rule 1 either $\sigma_{1} \cdot \tau_{1} \leq 1$ or $\sigma_{2} \cdot \tau_{2} \leq 1$ and we are done.

Suppose that $M\left(\sigma_{1}, \sigma_{2}\right)$ is irreducible.

3.10. Lemma. Either some component of $\delta_{1}$ compresses in $W_{1}\left(\sigma_{1}\right)$ or some component of $\delta_{2}$ compresses in $W_{2}\left(\sigma_{2}\right)$.

Proof. By 2.5 some component $c_{\sigma}$ of $\gamma$ compresses in $M\left(\sigma_{1}, \sigma_{2}\right)$. Let $D \subset$ $M\left(\sigma_{1}, \sigma_{2}\right)$ be a compressing disk for $c_{\sigma}$, chosen so as to minimize the number of components of intersection with the splitting disk $E$ in $M \subset M(\sigma)$. Then a standard innermost disk argument shows that $D \cap E$ consists only of arcs. If $D \cap E=\varnothing$, then $c_{\sigma} \cap \partial E=\varnothing$, so $c_{\sigma}$ is either in $\delta_{1}$ or $\delta_{2}$, proving the lemma. If $D \cap E \neq \varnothing$, an outermost arc $\alpha$ of $d_{\sigma} \cap E$ in $D$ cuts off from $\partial D=c_{\sigma}$ an arc $\beta$ so that $\beta$ is essential in either $\left(F_{1}, \partial E\right)$ or $\left(F_{2}, \partial E\right)$, say the former. Then $\alpha \cup \beta$ is in $\delta_{1}$ and the subdisk of $D$ which $\alpha$ cuts off is a compressing disk for $\alpha \cup \beta$ in $W_{1}\left(\sigma_{1}\right)$.

To resume the proof of Theorem 3.9 we will assume without loss of generality that some component of $\delta_{1}$ compresses in $W\left(\sigma_{1}\right)$.

If there is a slope $\tau_{1}$ so that $W_{1}\left(\tau_{1}\right)$ is reducible (so Rule 1 applies), then $\sigma_{1} \cdot \tau_{1} \leq 1$ by [Sc3, 6.1]. If there is no such slope, so Rule 2 applies, then some (possibly different) component $c_{\tau}$ of $\delta_{1}$ compresses in $W_{1}\left(\tau_{1}\right)$. Since $c_{\sigma}$ and $c_{\tau}$ are both in $\delta_{1}, c_{\sigma} \cdot c_{\tau}=1$. Then, by 3.7, $\sigma_{1} \cdot \tau_{1}=1$.

General case. If $(M, \gamma)$ is not itself a $J$-cobordism, then construct a taut sutured manifold hierarchy

$$
(M, \gamma) \stackrel{S_{1}}{\longrightarrow}\left(M_{1}, \gamma_{1}\right) \stackrel{S_{2}}{\longrightarrow} \cdots \stackrel{S_{i}}{\longrightarrow}\left(M_{i}, \gamma_{i}\right) \stackrel{s_{i+1}}{\longrightarrow} \ldots
$$

so that each $S_{i}$ is disjoint from $T=T_{1} \cup T_{2}$. By [ $\mathrm{Scl}, 2.6$ and 4.17] such a sequence eventually terminates in a taut sutured manifold $\left(M_{n}, \gamma_{n}\right)$ with $H_{2}\left(M_{n}, \partial M_{n}-T\right)=0$, i.e., $M_{n}$ is a $J$-cobordism on $T$. Moreover, $\left(M\left(\sigma_{1}, \sigma_{2}\right), \gamma\right)$ is taut if $\left(M_{n}\left(\sigma_{1}, \sigma_{2}\right), \gamma_{n}\right)$ is taut. The proof now follows by applying the special case to $\left(M_{n}\left(\sigma_{1}, \sigma_{2}\right), \gamma_{n}\right)$. 


\section{REFERENCES}

[B] H. Bass, J. Birman, and J. Morgan, The Smith conjecture, Academic Press, 1984.

[BS] S. Bleiler and M. Scharlemann, Tangles, Property P, and a problem of J. Martin, Math. Ann. 273 (1986), 215-225.

[Bo] F. Bonahon, Involutions et fibrés de Seifert dans les variétés de dimension 3, Thèse de $3 \mathrm{e}$ cycle, Orsay, 1979.

[BZ] G. Burde and H. Zieschang, Knots, de Gruyter Stud. Math., vol. 5, de Gruyter, 1985.

[Ga] D. Gabai, Foliations and the topology of 3-manifolds, J. Differential Geom. 18 (1983), 445-503.

[GL] C. Gordon and J. Luecke, Reducible manifolds and Dehn surgery (to appear).

[Li] W. B. R. Lickorish, The unknotting number of a classical knot, Contemp. Math., vol. 44, Amer. Math. Soc., Providence, R.I., 1985, pp. 117-121.

[Mo] J. Montesinos, Surgery on links and double branched coverings of $S^{3}$, Ann. of Math. Stud., no. 84, Princeton Univ. Press, Princeton, N.J., 1975, p. 227-259.

[Sc1] M. Scharlemann, Sutured manifolds and generalized Thurston norms, J. Differential Geom. 29 (1989), 557-614.

[Sc2] _ Handlebody complements in the 3-sphere, Proc. Amer. Math. Soc. 115 (1992), 11151117.

[Sc3] __, Producing reducible 3-manifolds by surgery on a knot, Topology 29 (1990), 481-500.

[ST] M. Scharlemann and A. Thompson, Link genus and the Conway moves, Comment. Math. Helv. 64 (1989), 527-535.

[Th] W. Thurston, Norm on the homology of three-manifolds, Mem. Amer. Math. Soc., No. 339 (1986), 99-130.

Department of Mathematics, University of California, Santa Barbara, California 93106

E-mail address: mgscharl@math.ucsb.edu; ma00mgs@ucsbuxa.ucsb.edu 